\title{
Herausforderung an die Ärztliche Krankenhausführung
}

Brunkhorst/Stockhorst: Aktuelle Herausforderungen an die Ärztliche Krankenhausführung. Herausgegeben von Prof. Dr. med. Reinhard Brunkhorst und Dr. med. Hermann Stockhorst. MBA-2007. XII 309 Seiten. Gebunden. $€ 68,-$. ISBN 978-3-87081-510-3. Verlagsgruppe Hüthig Jehle Rehm

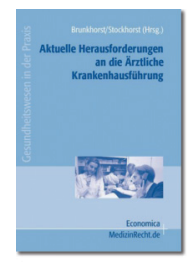

Der vorliegende Band enthält 16 Beiträge zum Thema. Es ist also ein sogenanntes Vielmännerbuch. Die unterschiedlichsten Aspekte der ärztlichen Krankenhausführung werden besprochen. Beispielsweise der Einsatz von Drittmittel, Arbeitsrecht, Führungsstrukturen, Gesundheitspolitik, Krankenhausorganisation, etc. Es soll gezeigt werden, wie man sich zukünftigen, vorwiegend wirtschaftlichen Herausforderungen stellen soll und wie man Lösungen entwickeln kann.

Die dort behandelten Themen lagen einer Veranstaltungsreihe zugrunde, die sich unter dem Namen „Herausforderung an die Ärztliche Krankenhausführung“ an Ärzte im Krankenhausmanagement, an Ärztliche Direktoren usw. richtete. In der Einleitung steht, dass die Durchsetzungsfähigkeit des ärztlichen Krankenhausmanagements gegenüber den Chefärzten und den klinischen Abteilungsleitern garantiert werden muss. Grundsätzlich wird offenbar davon ausgegangen, dass zwischen ärztlichem Krankenhausmanagement und Chefärzten eine Kluft besteht. Dabei ist nicht zu übersehen, dass es ein ärztliches Krankenhausmanage- ment noch nicht überall gibt, bisher eigentlich nur in sogengannten privat strukturierten Krankenanstalten.

Diese Kluft wird in dem vorliegenden Buch offensichtlich so verstanden, dass Chefärzte von vornherein nicht in der Lage sind, wirtschaftlich zu denken und einer kaufmännischen Vernunft zum Siege zu verhelfen. Diese Tendenz ist mehr oder weniger in allen Beiträgen zu finden, besonders eklatant in dem Beitrag von B. Füllekrug: „Strukturwandel und Paradigmenwechsel im Gesundheitswesen; das deutsche Chefarztsystem vor seiner Herausforderung“.

Selbstverständlich setzt der Autor voraus, dass das Ordinariat und Chefarztsystem überholt, total veraltet und sanierungsbedürftig ist. Dass das bisherige System immerhin dazu geführt hat, dass das deutsche Gesundheitswesen noch im vorderen Feld weltweit im Vergleich mit anderen Industrienationen angesiedelt ist, wird ignoriert. Selbstverständlich wird als erstes festgestellt, dass die strenge Hierarchie (gibt es diese strenge Hierarchie eigentlich noch, oder rennt man offene Türen ein?) die Ursache allen Übels darstellt. Es gibt aber kaum eine medizinische Disziplin, die sich inzwischen nicht so aufgesplittert hätte, dass noch von einem allmächtigen Chefarzt die Rede sein kann.

Der Hinweis auf das Gesundheitswesen in anderen Ländern verfängt nicht. Hier sind Ärzte bekannt, die aus den gelobten Ländern wieder zurückgekehrt sind, vor allem deswegen, weil inzwischen hier in Deutschland Plätze frei geworden sind und hier zu Lande immer noch die Arbeit besser honoriert wird als anderswo. Wenn ein Land eine funktionierende Medizinerausbildung ausweist und andere Länder sich diese Mediziner holen wollen, ist das doch ein Zeichen dafür, dass dieses System nicht so schlecht sein kann, wie es zur Zeit gemacht wird. Wir sind auf dem typisch deutschen Wege, wie üblich das Kind mit dem Bade auszuschütten und es ist nur zu hoffen, dass diese Tendenz möglichst schnell als zumindest übertrieben und in der Praxis als unbewiesen erkannt wird. Erinnert sei in diesem Zusammenhang an die These, dass nur die zu quasi revolutionären Änderungen bereit sind, die zu dumm sind, kleinschrittig zu reformieren. Prof. F. Reimer, Weinsberg 